\title{
Procedure Dose Description
}

National Cancer Institute

\section{Source}

National Cancer Institute. Procedure Dose Description. NCI Thesaurus. Code C117519.

A textual description of the agent dose level(s) or range(s) for the procedure per subject, according to the study protocol. 